\title{
Chemokines in the cerebrospinal fluid of patients with active and stable relapsing-remitting multiple sclerosis
}

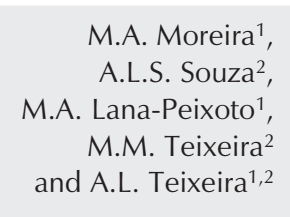

${ }^{1}$ Centro de Investigação em Esclerose Múltipla, Hospital das Clínicas, ${ }^{2}$ Laboratório de Imunofarmacologia, Departamento de Bioquímica e Imunologia, Instituto de Ciências Biológicas, Universidade Federal de Minas Gerais, Belo Horizonte, MG, Brasil

\section{Correspondence}

A.L. Teixeira

Laboratório de Imunofarmacologia

Departamento de Bioquímica e

Imunologia, ICB, UFMG

Avenida Antônio Carlos, 6627

Bloco O4, Sala 202

31270-901 Belo Horizonte, MG

Brasil

Fax: +55-31-3499-2651

E-mail: altexjr@hotmail.com

Received April 8, 2005 Accepted December 14, 2005

\begin{abstract}
Multiple sclerosis (MS) is a chronic inflammatory demyelinating disease of the human central nervous system. Although its etiology is unknown, the accumulation and activation of mononuclear cells in the central nervous system are crucial to its pathogenesis. Chemokines have been proposed to play a major role in the recruitment and activation of leukocytes in inflammatory sites. They are divided into subfamilies on the basis of the location of conserved cysteine residues. We determined the levels of some CC and CXC chemokines in the cerebrospinal fluid (CSF) of 23 relapsing-remitting MS patients under interferon- $\beta-1 \mathrm{a}$ therapy and 16 control subjects using ELISA. MS patients were categorized as having active or stable disease. CXCL10 was significantly increased in the CSF of active MS patients (mean \pm SEM, $369.5 \pm 69.3 \mathrm{pg} / \mathrm{mL}$ ) when compared with controls $(178.5 \pm$ $29.1 \mathrm{pg} / \mathrm{mL}, \mathrm{P}<0.05)$. CSF levels of CCL2 were significantly lower in active MS $(144.7 \pm 14.4 \mathrm{pg} / \mathrm{mL})$ than in controls $(237.1 \pm 16.4 \mathrm{pg} /$ $\mathrm{mL}, \mathrm{P}<0.01)$. There was no difference in the concentration of CCL2 and CXCL10 between patients with stable MS and controls. CCL5 was not detectable in the CSF of most patients or controls. The qualitative and quantitative differences of chemokines in CSF during relapses of MS suggest that they may be useful as a marker of disease activity and of the mechanisms involved in the pathogenesis of the disease.
\end{abstract}

Key words

- CXCL10

- CCL2

- Inflammation

- Multiple sclerosis

- Leukocyte recruitment

\section{Introduction}

Multiple sclerosis (MS) is a chronic inflammatory disease of the human central nervous system (CNS) resulting in the formation of plaques of demyelination. Although the cause of MS is unknown, it is generally considered to be an autoimmune disease mediated by $\mathrm{CD}^{+}{ }^{+} \mathrm{T}$ cells, which produce high levels of type 1 cytokines, such as interferon- $\gamma(1,2)$. These Th1 cells initiate an inflammatory process, recruiting and activating circulatory monocytes as well as microglia cells, which destroy myelin. Thus inflammation, mainly consisting of $\mathrm{T}$ cells and activated macrophages, seems to play a critical role in the pathogenesis of MS $(3,4)$.

Chemokines are low-molecular weight 
cytokines that mediate the migration of leukocytes to inflammatory sites $(5,6)$. They are divided into subfamilies $(\mathrm{CC}, \mathrm{CXC}, \mathrm{XC}$, CX3C) based on the location of conserved cysteine residues. Since mononuclear cell recruitment and activation in the CNS are fundamental steps in the pathogenesis of MS, chemokines have been proposed to play a major role in this autoimmune demyelinating process (7). Previous studies have demonstrated that in MS CXCL10 and CCL5 are increased in the cerebrospinal fluid (CSF) of patients with MS compared with control subjects, while CCL2 is significantly decreased (7-10). There is a growing body of evidence suggesting that these altered CSF chemokine levels could be used as surrogate markers of MS disease activity (9).

In the present study, we evaluated the CSF levels of CC and CXC chemokines from active and stable relapsing-remitting MS (RR-MS) patients and control subjects. This is the first study to evaluate chemokines in Brazilian MS patients.

Table 1. Baseline demographic and clinical characteristics of the subjects studied.

\begin{tabular}{lccc}
\hline & \multicolumn{2}{c}{ MS patients } & $\begin{array}{c}\text { Control group } \\
(\mathrm{N}=16)\end{array}$ \\
\cline { 2 - 4 } & Active MS $(\mathrm{N}=11)$ & Stable MS $(\mathrm{N}=12)$ & \\
\hline Age & $34.6 \pm 8.9(21-48)$ & $35.0 \pm 9.7(20-47)$ & $39.5 \pm 10.3(24-52)$ \\
Gender $(\mathrm{M} / \mathrm{F})$ & $1 / 10$ & $1 / 11$ & $4 / 12$ \\
EDSS & $4.9 \pm 1.2(2-6.5)$ & $3.0 \pm 1.5(1-4)$ & -
\end{tabular}

Data are reported as means \pm SEM (range) except for gender. MS = multiple sclerosis; $\mathrm{M} / \mathrm{F}=$ male/female; EDSS = Expanded Disability Status Scale score.

Figure 1. CXCL10 levels in cerebrospinal fluid (CSF) of patients with active multiple sclerosis (MS; $N=11)$, stable MS $(N=12)$ and control subjects $(\mathrm{N}=16) .{ }^{*} \mathrm{P}$ $<0.05$ compared to control (Newman-Keuls multiple comparison post-test).

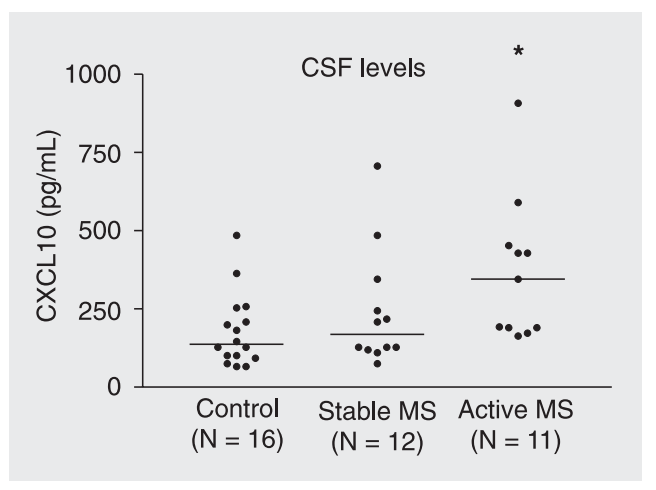

\section{Subjects and Methods}

\section{Subjects}

All subjects (patients and controls) enrolled in this study were from Belo Horizonte, State of Minas Gerais, Brazil, where the prevalence of MS is about 15 cases per 100,000 persons (11). Twenty-three patients diagnosed as having clinical definite RRMS according to Poser's criteria (12) were studied (Table 1). Twelve MS patients were clinically stable under interferon- $\beta-1 \mathrm{a}$ therapy (mean score on the Expanded Disability Status Scale (EDSS) $3.0 \pm 1.5$ ). The remaining 11 patients had active disease (mean EDSS $4.9 \pm 1.2$ ). Disease activity was defined according to clinical criteria, i.e., patients were in relapse with the presence of new neurological symptoms or deterioration of previous ones. Accordingly, the EDSS scores of patients with active RR-MS were higher than those of patients with stable RRMS $(\mathrm{P}<0.05)$. Active MS patients were also under interferon- $\beta-1$ a therapy and the CSF was collected before any steroid therapy.

Sixteen individuals with no neurological disease undergoing lumbar puncture as part of an anesthetic procedure for surgical intervention (orthopedic and gynecologic) were recruited as the control group. The absence of neurological disease was established on the basis of clinical history and neurological examination of the subjects. This group was matched with MS patients for age and gender.

CSF samples were collected aseptically and stored at $-70^{\circ} \mathrm{C}$ until required. The study was approved by the Ethics Committee of Universidade Federal de Minas Gerais and all subjects gave written informed consent to participate.

\section{Chemokine analysis}

The concentration of chemokines in the CSF of MS patients and controls was measured by sandwich ELISA with matched anti- 
body pairs for CXCL10 (formerly $\gamma$-interferon inducible protein of $10 \mathrm{kDa}$; Pharmingen, San Diego, CA, USA), and with ELISA kits for CCL2 (formerly monocyte chemotactic protein-1) and CCL5 (formerly regulated upon activation of normal $\mathrm{T}$ cell expressed and secreted; R\&D Systems, Minneapolis, MN, USA). All samples were used undiluted and assayed in duplicate in the same plate. The intra-assay variation was less than $5 \%$. The lower detection limits of the assays were $5 \mathrm{pg} / \mathrm{mL}$ for CCL2 and CCL5, and 10 $\mathrm{pg} / \mathrm{mL}$ for CXCL10.

\section{Statistical analysis}

Differences among groups were determined by analysis of variance (ANOVA) and the Newman-Keuls multiple comparison post-test, with the level of significance set at $\mathrm{P}<0.05$. All calculations were performed using Instat software (GraphPad, San Diego, CA, USA).

\section{Results}

CXCL10 levels were significantly higher in CSF of active RR-MS patients (mean \pm SEM, $369.5 \pm 69.3 \mathrm{pg} / \mathrm{mL}$; median (25th75th percentile), 345.4 (190.7-521.6) pg/ $\mathrm{mL})$ when compared with controls (178.5 \pm $29.1 \mathrm{pg} / \mathrm{mL} ; 137.0$ (97.1-231.3) pg/mL; $\mathrm{P}<$ 0.05; Figure 1). Mean CXCL10 value for stable RR-MS was $241.5 \pm 54.1 \mathrm{pg} / \mathrm{mL}$ and did not differ significantly from control or active RR-MS values.

CCL2 levels were significantly lower in CSF of active RR-MS patients (mean \pm SEM, $144.7 \pm 14.4 \mathrm{pg} / \mathrm{mL}$; median (25th-75th percentile), 125.1 (105.8-202.2) pg/mL) when compared with controls $(237.1 \pm 16.4 \mathrm{pg} /$ $\mathrm{mL} ; 240.7$ (202.2-260.0) pg/mL; P < 0.01; Figure 2). The mean CCL2 value for stable RR-MS was $202.9 \pm 24.0 \mathrm{pg} / \mathrm{mL}$ and was not statistically different from control or active RR-MS values.

CCL5 was detected in only one patient with RR-MS and in one control subject, with no difference between them.

\section{Discussion}

Chemokines are involved in physiologic and pathologic leukocyte trafficking and have been investigated in several inflammatory diseases of the CNS $(8,9)$. In the present study, we evaluated CSF levels of CXCL10, CCL2 and CCL5 in active RR-MS and stable RR-MS patients and in individuals with no neurological disorders.

The CSF levels of CXCL10 were significantly increased in active RR-MS patients compared with control subjects. In contrast, the CSF levels of CCL2 were lower in patients with active RR-MS than in controls. The levels of both CCL2 and CXCL10 in stable RR-MS patients were similar to those of controls and active RR-MS patients. This is the first study to report levels of chemokines in CSF of MS patients in Brazil and in the Southern Hemisphere. The present results demonstrate that there is a similar increase in the concentration of CXCL10 as well as a decrease in the concentration of CCL2 in Brazilian patients, as also reported for European/North American patients (10, 13-17). Thus, although the genetic background of our population is different and the prevalence of the disease is lower than in European countries or in the United States $(11,18,19)$, the pattern of CSF levels of CXCL10 and CCL2 is similar. This is consistent with a related pathogenetic mechan-

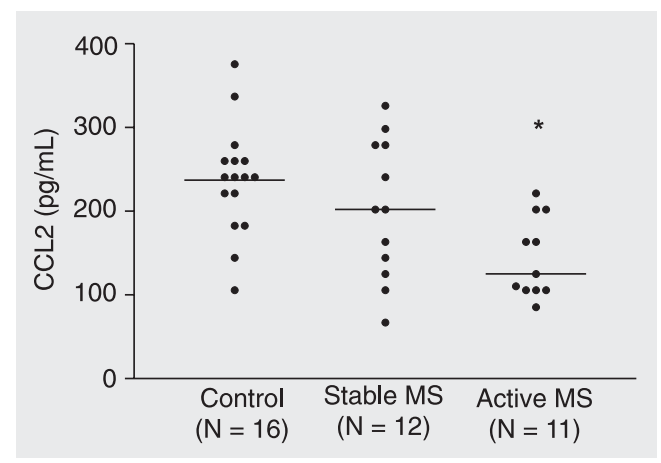

Figure 2. CCL2 levels in cerebrospinal fluid (CSF) from patients with active multiple sclerosis (MS; $N=11)$, stable $M S(N=$ 12) and control subjects ( $N=$ 16). ${ }^{*} P<0.05$ compared to control (Newman-Keuls multiple comparison post-test). 
ism for the disease in these different populations.

Several studies have now shown that CXCL10 may activate CXCR3 to induce the migration and activation of Th1 lymphocytes and monocytes, cells relevant for MS pathogenesis (6). The finding of increased CXCL10 in CSF of patients with active disease is consistent with current paradigms that MS is a predominantly Th1 disease $(1,2)$. Studies of experimental autoimmune encephalomyelitis suggest a pathogenic role for CCL2 $(20,21)$. This is reinforced by the ability of CCL2 to induce the migration of mononuclear cells into the CNS and the presence of CCL2 at sites of demyelination in patients with MS (20,21). However, CCL2 may also have a role in facilitating $\mathrm{Th} 2$ responses under certain circumstances $(5,6)$. In this regard, the lower concentrations of CCL2 in CSF of patients with active MS may be due to the sequestration of the chemokine within MS lesions or, alternatively, may be a consequence of the altered Th1/Th2 balance observed in MS patients (20). The levels of both chemokines are consistent with their role in disease pathogenesis and in mediating leukocyte recruitment and the Th1/ Th2 cytokine balance.

In the present study, only two samples had detectable levels of CCL5. Thus, no difference could be established between groups. There are discordant results in the literature concerning CSF CCL5 levels in MS. Some studies failed to find any difference among groups $(14,15)$, while others demonstrated increased CSF levels of CCL5 during MS relapses $(10,22,23)$. The fact that the CSF concentration of this chemokine is very low and near the lower limit of detection by ELISA may explain these discrepant results.

In the present study, all patients were using interferon- $\beta$ - 1 a therapy at the time the samples were collected. At least one study has demonstrated that such therapy fails to modify the expression of chemokines (13). This is an interesting finding since chemokines may play a role in the disease and lack of interference with the production of these mediators may be one of the reasons why this therapy only partially ameliorates progression in only some of the treated patients (4).

The results of the present study support the view that CXCL10 and CCL2 are differently released during relapses of MS. There appears to be a profile of secretion compatible with an enhanced Th1 response. Moreover, it appears that levels of chemokines in CSF may be useful as markers of disease activity (24).

\section{References}

1. Lassmann H \& Ransohoff RM (2004). The CD4-Th1 model for multiple sclerosis: a crucial re-appraisal. Trends in Immunology, 25: 132-137.

2. Hafler DA (2004). Multiple sclerosis. Journal of Clinical Investigation, 113: 788-794.

3. Kornek B \& Lassmann H (2003). Neuropathology of multiple sclerosis. Brain Research Bulletin, 61: 321-326.

4. Noseworthy JH, Lucchinetti C, Rodriguez M et al. (2000). Multiple sclerosis. New England Journal of Medicine, 343: 938-952.

5. Mackay CR (2001). Chemokines: immunology's high impact factors. Nature Immunology, 2: 95-101.

6. Moser B, Wolf M, Walz A et al. (2004). Chemokines: multiple levels of leukocyte migration control. Trends in Immunology, 25: 75-84.

7. Trebst C \& Ransohoff RM (2001). Investigating chemokines and chemokine receptors in patients with multiple sclerosis: opportuni- ties and challenges. Archives of Neurology, 58: 1975-1980.

8. Ransohoff RM (2002). The chemokine system in neuroinflammation: an update. Journal of Infectious Diseases, 186 (Suppl 2): S152S156.

9. Sellebjerg F \& Sorensen TL (2003). Chemokines and matrix metalloproteinase-9 in leukocyte recruitment to the central nervous system. Brain Research Bulletin, 61: 347-355.

10. Sorensen TL, Tani M, Jensen J et al. (1999). Expression of specific chemokines and chemokine receptors in the central nervous system of multiple sclerosis patients. Journal of Clinical Investigation, 103: 807-815.

11. Lana-Peixoto MA, Frota E, Campos GB et al. (2002). The prevalence of multiple sclerosis in Belo Horizonte, Brazil. Multiple Sclerosis, 8 (Suppl 1): 38-39.

12. Poser CM, Paty DW, Scheinberg L et al. (1983). New diagnostic 
criteria for multiple sclerosis: guidelines for research protocols. Annals of Neurology, 13: 227-231.

13. Franciotta D, Martino G, Zardini E et al. (2001). Serum and CSF levels of MCP-1 and IP-10 in multiple sclerosis patients with acute and stable disease and undergoing immunomodulatory therapies. Journal of Neuroimmunology, 115: 192-198.

14. Sindern E, Niederkinkhaus Y, Henschel M et al. (2001). Differential release of $B$-chemokines in serum and CSF of patients with relapsing-remitting multiple sclerosis. Acta Neurologica Scandinavica, 104: 88-91.

15. Mahad DJ, Howell SJ \& Woodroofe MN (2002). Expression of chemokines in the CSF and correlation with clinical disease activity in patients with multiple sclerosis. Journal of Neurology, Neurosurgery, and Psychiatry, 72: 498-502.

16. Scarpini E, Galimberti D, Baron P et al. (2002). IP-10 and MCP-1 levels in CSF and serum from multiple sclerosis patients with different clinical subtypes of the disease. Journal of Neurological Sciences, 195: 41-46.

17. Narikawa K, Misu T, Fujihara K et al. (2004). CSF chemokine levels in relapsing neuromyelitis optica and multiple sclerosis. Journal of Neuroimmunology, 149: 182-186.

18. Lana-Peixoto MA \& Lana-Peixoto MI (1992). Is multiple sclerosis in
Brazil and Asia alike? Arquivos de Neuropsiquiatria, 50: 119-125.

19. Arruda WO, Scola RH, Teive HA et al. (2001). Report on 200 cases from Curitiba, Southern Brazil and comparison with other Brazilian series. Arquivos de Neuropsiquiatria, 59: 165-170.

20. Mahad DJ \& Ransohoff RM (2003). The role of MCP-1 (CCL2) and CCR2 in multiple sclerosis and experimental autoimmune encephalomyelitis (EAE). Seminars in Immunology, 15: 23-32.

21. Santos AC, Barsante MM, Arantes RM et al. (2005). CCL2 and CCL5 mediate leukocyte adhesion in experimental autoimmune encephalomyelitis: an intravital microscopy study. Journal of Neuroimmunology, 162: 122-129.

22. Bartosik-Psujek H, Belniak E, Mitosek-Szewczyk K et al. (2004). Interleukin-8 and RANTES levels in patients with relapsing-remitting multiple sclerosis treated with cladribine. Acta Neurologica Scandinavica, 109: 390-392.

23. Bartosik-Psujek H \& Stelmasiak $Z$ (2005). The levels of chemokines CXCL8, CCL2 and CCL5 in multiple sclerosis patients are linked to the activity of the disease. European Journal of Neurology, 12: 4954.

24. Bielekova B \& Martin R (2004). Development of biomarkers in multiple sclerosis. Brain, 127: 1463-1478. 